\title{
PROGRAMA DE PROMOCIÓN DE LA SALUD Y EDUCACIÓN EN VALORES A TRAVÉS DEL CINE Y EL VIDEO EN LA COMUNIDAD AUTÓNOMA DE LA RIOJA (1995-2012)
}

\author{
Purificación Echeverría Cubillas \\ $\mathbf{M}^{\mathrm{a}}$ Luisa Rodríguez Moroy \\ Consejería de Salud y Servicios Sociales, Gobierno de La Rioja
}

RESUMEN: En este trabajo se describe el Programa de Promoción de la Salud y Educación en Valores a través del cine y video realizado con alumnos de $4^{\circ}$ de Educación Secundaria Obligatoria (ESO) y $1^{\circ}$ de Programas de Cualificación Profesional Inicial (PCPI) de la Comunidad Autónoma de La Rioja durante los años 1995-2012.

El cine puede ser utilizado como un importante recurso didáctico a la hora de llevar a cabo la Promoción y Educación para la salud en el medio escolar.

La participación de los centros en el programa ha permanecido estable a lo largo de los años, con variaciones mínimas, entre el 70 Y el $80 \%$; el programa está bien valorado por el profesorado; también los alumnos realizan una valoración positiva del mismo; se destaca el alto porcentaje de alumnos, entre el 80 y $90 \%$, que señalan la utilidad de las actividades realizadas, posteriores al visionado, para generar reflexión.

PALABRAS CLAVE: Estudiantes de Secundaria, promoción y educación para la salud, películas y videos, profesorado.

\section{PROGRAM OF HEALTH PROMOTION AND VALUES EDUCATION THROUGH FILM AND VIDEO IN LA RIOJA (1995-2012)}

ABSTRACT: In this paper we describe the Program for Promotion of Health and Education in Values through Film and Video, aimed at the students of 4th of Secondary Education (ESO) and of 1st of Professional Qualification Programs (PCPI) in the Autonomous Community of La Rioja during the period of 1995-2012. Its main assumption is that movies can be used as an important educational resource to promote health culture in schools. 
The main outputs of the program were that the participation of schools remained stable over the years, with minor variations between the 70/80\%, and that the program was well received by teachers and students, who made a positive assessment thereof. Besides, it must be highlighted the high percentage of students (between 80/90\%) indicating the usefulness of the activities carried out to generate reflection after watching the movies.

KEYWORDS: High school students, promotion and health education, movies and videos, teaching staff.

Recibido: 10/07/2012

Aceptado: 21/09/2013

\section{INTRODUCCIÓN}

La Organización Mundial de la Salud en su Carta Magna (1946) define la salud como "completo estado de bienestar físico, psíquico y social". Esta definición formula la salud en términos positivos y no solo como ausencia de enfermedad. En su dimensión individual, la Promoción de la Salud ${ }^{1}$ constituye un proceso que permite a las personas el desarrollo de las habilidades necesarias para aumentar el control sobre su salud adoptando estilos de vida saludables, incrementando y reforzando sus recursos personales mediante la ampliación de la información, la sensibilización, el desarrollo de una percepción crítica sobre los condicionantes del entorno que afectan a la salud, la educación y el entrenamiento en habilidades para la vida. Para concretar las actuaciones de este nivel individual, la Promoción de la Salud utiliza la Educación para la Salud y el fomento de entornos saludables.

Los alumnos de segundo ciclo de la ESO se encuentran en plena adolescencia; periodo que comienza con los cambios fisiológicos de la pubertad y finaliza a los 20 años (OMS). Se trata de una etapa de transición en la que no se es niño, pero en la que aún no se tiene el estatus de adulto. Es lo que Erikson² denominó una "moratoria social", un compás de espera que la sociedad da a sus miembros jóvenes mientras se preparan para ejercer los roles adultos. Constituye una de las etapas más vulnerables en el desarrollo de la personalidad del individuo; los adolescentes adquieren una mayor autonomía e independencia (manejo de dinero, salidas con amigos, fiestas, decisiones respecto a los estudios...), se "distancian" de la familia y se hacen más dependientes del grupo de amigos, también experimentan un importante desarrollo corporal, emocional e intelectual. Todos estos factores, unidos a su baja percepción de riesgo, les hace muy vulnerables respecto a conductas tales como el consumo de drogas, relaciones sexuales precoces y sin medidas de prevención, conducción temeraria, problemas de acoso escolar y violencia, etc.

La educación para la salud definida por Lawrence W. Green ${ }^{3}$ como "combinación de experiencias de aprendizaje planificadas, destinada a facilitar cambios voluntarios

1. Green, L. W.; Wilson A. L. y Lovato, C. Y. (1986). What changes can health promotion achieve and how long do these changes last? The tradeoffs between expediency and durability. Preventive Medicine, 15, 508-521.

2. Erikson, E. (1972). Sociedad y Adolescencia. Buenos Aires: Paidós.

3. Rochon, A. (1990). Educación para la Salud. Barcelona: Masson. 
de comportamiento saludable", puede ser una estrategia eficaz con la que abordar la promoción de la salud en los adolescentes.

El cine puede ser utilizado como un importante recurso didáctico, ya que nos permite informar y formar de forma distendida y lúdica; nos ofrece la posibilidad de utilizar las películas como instrumentos para educar porque nos proporciona modelos, puede ayudar a cambiar actitudes, enseña estilos de vida, propicia la reflexión y es, además, un importante elemento motivador.

El cine posee potencial educativo para ${ }^{4}$ :

- Aportar conocimientos: historia y costumbres, arte, música, valores, relaciones humanas.

- Posibilitar el desarrollo de la sensibilidad, por su carácter visual y sonoro, desarrollando la observación y la percepción sensorial. Nos permite, asimismo, trabajar de forma conjunta las ideas y emociones.

- Desarrollar la capacidad cognitiva: percibir las distintas imágenes, diálogos y acciones de los personajes a través del tiempo; el cine requiere desarrollar la atención y la memoria, así como procesar las distintas informaciones, codificando y decodificando mensajes.

- Desarrollar la capacidad de comunicación: en el cine se emiten mensajes verbales y no verbales; mediante la realización de las actividades posteriores al visionado, los alumnos expresan, de forma oral y/o escrita, sus opiniones e ideas.

- Promover el pensamiento crítico: a través de la interpretación, el análisis y la valoración de las distintas situaciones, conflictos y relaciones que viven los personajes.

- Desarrollar actitudes y valores positivos, posibilitando mediante el análisis de las actitudes y conductas de los personajes la clarificación y asunción de los mismos.

El Currículo de Educación Secundaria Obligatoria (Decreto 5/2011, de 28 de enero, Boletín Oficial de La Rioja), establece el desarrollo de distintas capacidades, muchas de las cuales pueden desarrollarse y trabajarse a partir del visionado de distintas películas. Se destaca:

- Fomentar actitudes que favorezcan la convivencia y eviten la violencia en el ámbito escolar, familiar y social. (Objetivo de etapa).

- Conocer, asumir y ejercer sus derechos y deberes en el respeto a los demás, practicar la tolerancia, la cooperación y solidaridad entre las personas y los grupos, ejercitarse en el diálogo afianzando los derechos humanos como valores comunes de una sociedad plural, abierta y democrática. (Objetivo de etapa).

- Valorar la creación artística y comprender el lenguaje de las distintas manifestaciones artísticas, utilizando diversos medios de expresión y representación. (Objetivo de etapa).

4. Gobierno de La Rioja (2011). Programas de Educación para la Salud en Educación Secundaria. 
- Desarrollar hábitos favorables a la promoción de la salud personal y comunitaria, facilitando estrategias que permitan hacer frente a los riesgos de la sociedad actual en aspectos relacionados con la alimentación, el consumo, las drogodependencias y la sexualidad. (Objetivo Ciencias de la Naturaleza).

Asimismo, la ley de Salud de La Rioja (2/2002, de 17 de abril) indica, en su artículo 44, apartados 2 g) y 2 h) que el Sistema Público de Salud de La Rioja Ilevará a cabo las siguientes actuaciones:

- Promoción de hábitos de vida saludables en la población.

- Educación para la salud de la población, como elemento primordial para contribuir a la mejora de la salud individual y colectiva.

El Programa de Promoción de la salud y educación en valores a través del cine se puso en marcha, en Logroño, durante el curso 1995/96; inicialmente se proyectaban 5 películas; posteriormente, en función de los datos de la evaluación, se redujeron a 3 por curso escolar. En el curso 1996/97 el programa se ofertó a los municipios que disponían de sala de cine, contando para ello con la colaboración de los Ayuntamientos, participando, además de Logroño, Arnedo y Calahorra. Nájera se incorporó en el curso 1997/98, y en el siguiente (1998/99) los municipios de Haro y Santo Domingo. Por diversos motivos, la mayor parte de los Ayuntamientos, excepto el de Calahorra, se fueron, con el tiempo, "descolgando" del programa. Esta realidad, así como el hecho de tener que contar, para su realización, con importantes medios técnicos, no siempre disponibles en todos los municipios, hizo que se pusiera en marcha en el curso 1998/99 el Subprograma de Promoción de la salud y educación en valores a través del video, que básicamente consiste en proporcionar, en concepto de préstamo, a los centros inscritos en el mismo, las películas en formato video proyectadas el año anterior en el programa del cine, así como los cuadernos de cine correspondientes. En la actualidad, el Programa de Desarrollo personal y social a través del cine se desarrolla en Logroño, y el Subprograma de Desarrollo personal y social a través del video, se lleva a cabo en el resto de municipios de la Comunidad.

En el curso 2003/2004 se pone en marcha el Subprograma de cine dirigido a los padres y madres de los alumnos que participan en el mismo; la familia y los centros educativos comparten un objetivo común: la formación integral del adolescente, ambos aportarán los referentes que les permitan desarrollarse e integrarse en la sociedad. Estos sistemas de influencia necesitan converger para ganar en credibilidad y eficacia. El Programa de Desarrollo personal y social a través del cine posibilita que el adolescente reciba mensajes coherentes y educativos, de forma conjunta, desde el ámbito familiar y el educativo. Los temas que son objeto de reflexión y análisis en el aula, también lo son el ámbito familiar, ya que ambos han visionado la misma película. La sesión para los padres se proyecta en la misma semana que se proyecta para los alumnos, en horario distinto (tarde-noche). Después de su proyección se establece, entre los padres y el Psicólogo/pedagogo que modera la sesión, un coloquio sobre los aspectos que trata la película. También se les proporciona pautas educativas, así como una guía de la película específica para ellos.

El marco conceptual en el que se desarrolla este programa viene determinado, fundamentalmente, por las siguientes aportaciones: 
- La Teoría del Aprendizaje Social de Bandura 5 : las personas aprenden muchos de sus comportamientos y actitudes mediante la observación/imitación de las conductas de personas de su entorno: padres, hermanos, amigos, entorno social, mass-media.

- La Educación para la Salud ${ }^{6}$ : proceso que permite a las personas el desarrollo de habilidades necesarias para incrementar el control sobre su salud mediante las adopción de estilos de vida saludables, incrementando y reforzando sus recursos personales mediante la adquisición de conocimientos basados en la evidencia, el desarrollo de una percepción crítica referida a los condicionantes del entorno que afecta a su salud y el entrenamiento en habilidades para la vida.

Por ello, se plantea como objetivo general del Programa posibilitar, a través de un recurso motivador como es el cine, el desarrollo de capacidades y habilidades que favorezcan el desarrollo personal y social.

A su vez, este objetivo general se traduce en otros más específicos que contribuyen a su consecución. Son:

- Desarrollar capacidades personales que favorezcan la adquisición de estilos de vida saludables.

- Facilitar la clarificación y asunción de valores personales y sociales.

- Posibilitar la expresión de de ideas, emociones y experiencias.

- Desarrollar la reflexión y el análisis crítico respecto a las situaciones, conductas y actitudes de los distintos personajes.

- Favorecer la afición al cine como instrumento de enriquecimiento cultural y de utilización de su tiempo libre.

Los objetivos quedan reflejados en una serie de contenidos a trabajar a lo largo del Programa:

- Conductas de riesgo: consumo de drogas, relaciones sexuales sin medidas de protección, conducción bajo el consumo de drogas, no respeto de las indicaciones de seguridad, vulnerabilidad personal, percepción de riesgo.

- Toma de decisiones: elementos a considerar.

- Relaciones familiares. Comunicación, resolución de problemas y conflictos.

- La amistad. Relaciones de pareja. El apoyo y la ayuda mutua. Solidaridad.

- Metas y futuro. La importancia del esfuerzo y la constancia.

- Valores personales y sociales : coherencia.

- Responsabilidad y libertad. El respeto a las normas. Los derechos y los deberes de unos y otros.

- La multiculturalidad: tolerancia, respeto, aceptación, enriquecimiento mutuo.

5. Bandura, A. (1977). Social Learning Theory. Englewood Cliffs: Prentice Hall.

6. OMS (1998), Promoción de la Salud. Glosario. 
- La violencia. La gestión de los conflictos. La importancia del diálogo y la comunicación con los otros. El acoso escolar. Violencia de pareja.

- Autonomía personal, tolerancia a la frustración, el autoconcepto.

- Las nuevas tecnologías y sus riesgos

Las películas son seleccionadas de acuerdo a los criterios siguientes: adecuación al alumnado que van dirigidas, temáticas que abordan y calidad de las mismas (premios, crítica, etc.). La relación de películas proyectadas y los temas que abordan se indican en el Anexo 1.

\section{Proceso metodológico}

\section{Población}

Alumnado de $4^{\circ}$ de Educación Secundaria Obligatoria (ESO) y $1^{\circ}$ de Programas de Cualificación Profesional Inicial (PCPI). Inicialmente, cuando se puso en marcha el programa, la población se integraba por el alumnado de $2^{\circ}$ de Bachillerato Unificado Polivalente (BUP) y primer ciclo de Formación Profesional (FP).

\section{Estrategia de intervención}

Convocatoria: tanto el programa de cine como el subprograma de video son ofertados en septiembre a los centros educativos de la Comunidad Autónoma de La Rioja que imparten Educación Secundaria. Se proporciona información sobre las diferentes películas, una ficha de inscripción, así como la temporalización.

\section{Desarrollo del Programa}

Programa de desarrollo personal y social a través el cine

Dirigido a los centros de Logroño; se proyectan, en horario escolar, tres películas de actualidad, una cada trimestre; son seleccionadas por su relación con temas que inciden, fundamentalmente, en los estilos de vida de la juventud actual, utilizando el siguiente protocolo de actuación:

- Presentación de la película.

- Proyección de la película: acuden, a la Sala Gonzalo de Berceo, los alumnos con los profesores. Los centros que se inscriben en el subprograma del video la proyectan en el lugar que asignen, aula, sala de audiovisuales, salón de actos, etc.

- Trabajo en el aula: una vez realizado el visionado de cada película, los profesores, con el apoyo de los cuadernos de cine, trabajan, en su clase, los diferentes aspectos reflejados y seleccionados para su análisis y reflexión.

- En el año 2003/04 se amplió este programa a los padres cuyos hijos participan en el programa, proyectándose las mismas películas, en la misma fecha y en horario de tarde-noche, y realizándose un debate-coloquio conducido por un psicólogo o pedagogo; también se les proporcionaba una guía sobre la película que incluía pautas educativas. 
Subprograma de desarrollo personal y social a través del video

Se oferta a los Centros de Educación Secundaria del resto de la Comunidad Autónoma. A cada centro inscrito se le proporcionan, en concepto de préstamo, las películas que se proyectaron el año anterior en el programa del cine, así como los cuadernos de cine. Cada profesor recibe las películas en formato DVD, una cada trimestre.

\section{Cronograma}

La convocatoria se realiza en septiembre. El Programa se desarrolla en los meses de octubre a mayo, proyectándose una película cada trimestre del curso escolar. Finalmente, la evaluación se lleva a cabo en los meses de mayo-junio.

\section{Recursos}

\section{Humanos}

Un psicólogo y un pedagogo. Técnicos de la Sección de Educación para la Salud (Servicio de Promoción de Salud. Dirección General de Salud Pública y Consumo. Gobierno de La Rioja). Personal técnico para la proyección en la Sala Gonzalo de Berceo.

\section{Materiales}

Infraestructura: salas para proyectar películas, proyector de cine/video cañón y ordenador para realizar las presentaciones a los padres.

\section{Didácticos}

— "Cuadernos de cine para el profesor": cada película consta de: ficha técnica, datos artísticos, sinopsis, premios, objetivos, metodología y actividades para los alumnos.

- "Cuadernos de cine para el alumno": ficha técnica, datos artísticos, sinopsis, premios y actividades para los alumnos.

- "Cuadernos de cine para los padres": ficha técnica, datos artísticos, sinopsis, premios y pautas educativas.

\section{Evaluación}

A lo largo de estos años se ha realizado una evaluación de proceso: cada año al finalizar la actividad, se cumplimentan, para valorar los distintos aspectos del programa, dos cuestionarios, uno por parte del profesorado y otro por el alumnado. Se establecen como indicadores:

- Evolución de la participación de los centros.

- Valoración global del programa, realizada por el profesor. 
- Utilidad de las actividades propuestas, valoración realizada por los alumnos.

- Películas mejor valoradas por el profesor.

- Evolución participación padres.

\section{Resultados}

La Figura 1 refleja la evolución del porcentaje de participación de centros desde el curso 1997/98 hasta el curso 2011/12.

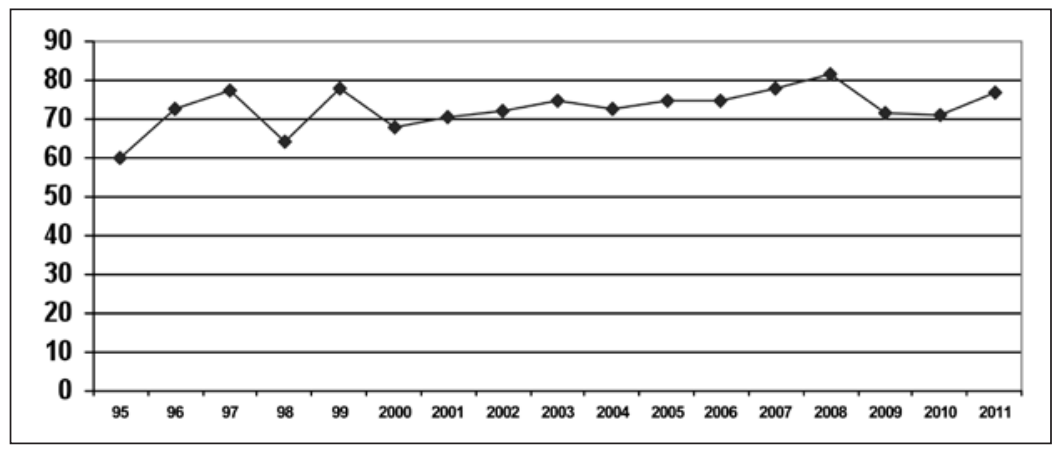

Figura 1. Evolución de la participación de los centros

Como puede observarse la participación es alta, manteniéndose estable a lo largo de los años, con variaciones mínimas, entre el 70 y el $80 \%$.

Asimismo, se detalla la valoración global del programa realizada por el profesorado (Tabla 1 ).

Tabla 1. Valoración global del profesorado

\begin{tabular}{|c|c|c|c|}
\hline \multirow{2}{*}{ Cursos } & \multicolumn{3}{|c|}{ Valoración } \\
\cline { 2 - 4 } & Muy adecuado & Adecuado & Inadecuado \\
\hline $1998 / 99$ & $11 \%$ & $82 \%$ & $7 \%$ \\
\hline $1999 / 00$ & $23 \%$ & $74 \%$ & $3 \%$ \\
\hline $2000 / 01$ & $40 \%$ & $56 \%$ & 0 \\
\hline $2001 / 02$ & $42 \%$ & $55 \%$ & $3 \%$ \\
\hline $2002 / 03$ & $47 \%$ & $53 \%$ & 0 \\
\hline $2003 / 04$ & $40 \%$ & $58 \%$ & 0 \\
\hline $2004 / 05$ & $57 \%$ & $43 \%$ & 0 \\
\hline $2005 / 06$ & $18 \%$ & $71 \%$ & 0 \\
\hline $2006 / 07$ & $44 \%$ & $56 \%$ & $4 \%$ \\
\hline $2007 / 08$ & $32 \%$ & $64 \%$ & 0 \\
\hline $2008 / 09$ & $38 \%$ & $62 \%$ & 0 \\
\hline $2009 / 10$ & $45 \%$ & $55 \%$ & 0 \\
\hline $2010 / 11$ & $50 \%$ & $50 \%$ & 0 \\
\hline $2011 / 12$ & $66 \%$ & $34 \%$ & 0 \\
\hline
\end{tabular}


La información de las películas mejor valoradas se obtiene a través del cuestionario cumplimentado por los profesores, preguntándoles su opinión sobre cuál es la película que más interesante ha resultado a los alumnos, así como con cuál piensan que se han sentido más identificados (Tabla 2).

Tabla 2. Películas mejor valoradas

\begin{tabular}{|l|l|}
\hline \multicolumn{1}{|c|}{ Curso } & \multicolumn{1}{c|}{ Películas mejor valoradas } \\
\hline $1998 / 99$ & El indomable Will Hunting \\
\hline $1999 / 00$ & American History X \\
\hline $2000 / 01$ & El Bola \\
\hline $2001 / 02$ & Espera el último baile \\
\hline $2002 / 03$ & Quiero ser como Beckham \\
\hline $2003 / 04$ & Planta $4^{\text {a }}$ \\
\hline $2004 / 05$ & Héctor y Thirteen \\
\hline $2005 / 06$ & Los chicos del coro \\
\hline $2006 / 07$ & Gol \\
\hline $2007 / 08$ & Diarios de la callle \\
\hline $2008 / 09$ & La Ola y Cobardes \\
\hline $2009 / 10$ & Ritmos de Barrio y el $1^{\text {er }}$ día del resto de tu vida \\
\hline $2010 / 11$ & Invictus \\
\hline $2011 / 12$ & Un sueño posible \\
\hline
\end{tabular}

El programa es valorado de forma positiva; la evaluación del profesor nos ha proporcionado valiosa información que ha servido para incrementar la calidad del programa.

La valoración realizada por los alumnos, respecto a la utilidad de las actividades propuestas en los Cuadernos de Cine, apunta a la ampliación de sus conocimientos, al fomento de la reflexión y el debate, así como a la participación (Tabla 3).

Tabla 3. Utilidad de las actividades según el alumnado

\begin{tabular}{|c|c|c|c|}
\hline & $\begin{array}{c}\text { Les ha servido } \\
\text { para ampliar } \\
\text { conocimientos }\end{array}$ & $\begin{array}{c}\text { Les ha servido para } \\
\text { reflexionar }\end{array}$ & $\begin{array}{c}\text { Les ha servido para } \\
\text { debatir y participar }\end{array}$ \\
\hline $1998 / 99$ & $68 \%$ & $90 \%$ & $85 \%$ \\
\hline $1999 / 00$ & $82 \%$ & $92 \%$ & $81 \%$ \\
\hline $2000 / 01$ & $73 \%$ & $39 \%$ & $76 \%$ \\
\hline $2001 / 02$ & $63 \%$ & $77 \%$ & $55 \%$ \\
\hline $2002 / 03$ & $75 \%$ & $96 \%$ & $96 \%$ \\
\hline $2003 / 04$ & $84 \%$ & $90 \%$ & $51 \%$ \\
\hline $2004 / 05$ & $64 \%$ & $87 \%$ & \\
\hline
\end{tabular}


Tabla 3. Utilidad de las actividades según el alumnado (continuación)

\begin{tabular}{|c|c|c|c|}
\hline & $\begin{array}{c}\text { Les ha servido } \\
\text { para ampliar } \\
\text { conocimientos }\end{array}$ & $\begin{array}{c}\text { Les ha servido para } \\
\text { reflexionar }\end{array}$ & $\begin{array}{c}\text { Les ha servido para } \\
\text { debatir y participar }\end{array}$ \\
\hline $2005 / 06$ & $81 \%$ & $92 \%$ & $82 \%$ \\
\hline $2006 / 07$ & $67 \%$ & $79 \%$ & $54 \%$ \\
\hline $2007 / 08$ & $64 \%$ & $83 \%$ & $71 \%$ \\
\hline $2008 / 09$ & $83 \%$ & $87 \%$ & $60 \%$ \\
\hline $2009 / 10$ & $63 \%$ & $90 \%$ & $71 \%$ \\
\hline $2010 / 11$ & $62 \%$ & $77 \%$ & $45 \%$ \\
\hline $2011 / 12$ & - & $63 \%$ & \\
\hline
\end{tabular}

Es de destacar el alto porcentaje de alumnos que señala la utilidad de las actividades propuestas para generar reflexión.

Respecto a la evolución de la participación de los padres, desde el curso 2003/04 hasta el curso 2011/12, expresado en frecuencia de participantes, se refleja (Figura 2) que ha ido disminuyendo de forma progresiva y acusada. El número de padres que asistieron el primer año fue de 216 y el último de 70. Además, han participado muy poco en el coloquio posterior a la proyección. A la vista de estos resultados se ha decidido no ofertarlo el próximo curso.

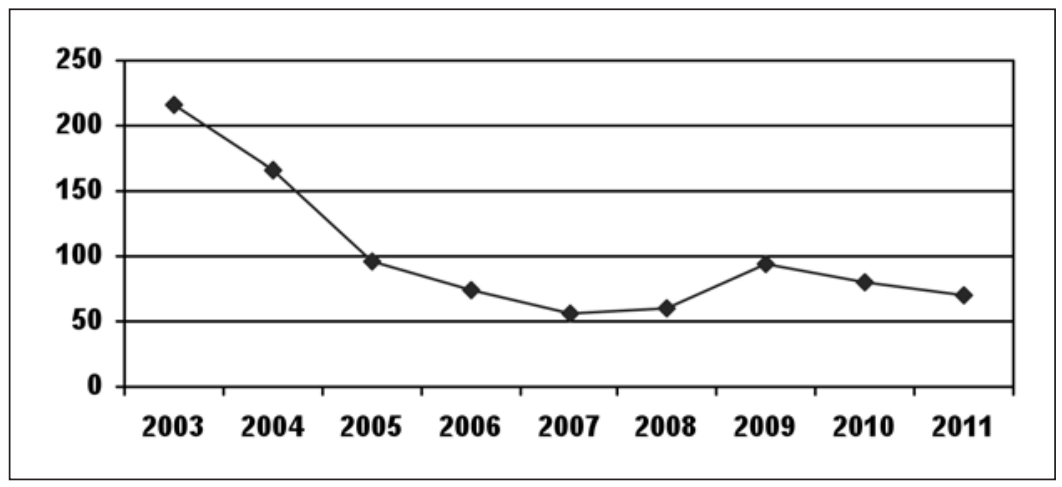

Figura 2. Evolución de la participación de los padres

\section{A MODO DE CONCLUSIÓN}

El cine puede ser utilizado como un importante recurso didáctico a la hora de Ilevar a cabo la Promoción y Educación para la salud en el medio escolar.

El programa de Promoción de la Salud y Educación en valores a través del cine y del vídeo está bien valorado por el profesorado y el alumnado de los centros. La participación en el mismo es alta (entre el 70 y el 80\%) manteniéndose estable a lo largo de los años; no así la de los padres, cuya baja participación determinó la supresión del mismo. 


\section{REFERENCIAS BIBLIOGRÁFICAS}

BANDURA, A. (1977). Social Learning Theory. Englewood Cliffs: Prentice Hall.

Decreto 5/2011, de 28 de enero, por el que se establece el Currículo de la Educación Secundaria Obligatoria de la Comunidad Autónoma de La Rioja.

ERIKSON, E. (1972). Sociedad y Adolescencia. Buenos Aires: Paidós.

GREEN L.W.; WILSON, A.L. y LOVATO, C.Y. (1986). What changes can health promotion and how long do these changes last? The tradeoffs between expediency and durability. Preventive Medicine, 15, 508-521.

Ley de Salud de La Rioja. Consejería de Salud y Servicios Sociales. Gobierno de La Rioja. 2003.

Organización Mundial de la Salud (1998). Promoción de la Salud. Glosario.

Gobierno de La Rioja (2011). Programas de Educación para la Salud en Educación Secundaria.

ROCHON, A. (1991). Educación para la Salud. Guía práctica para realizar un proyecto. Barcelona: Masson. 
